\title{
Dengue Incidence and the Prevention and Control Program in Malaysia
}

\author{
Rose Nani Mudin \\ Head of Vector Borne Disease Sector, Ministry of Health Malaysia
}

\begin{abstract}
The trend of dengue incidence in the regions and many countries has shown an increasing trend for the past few decades. The World Health Organization (WHO) reported that the incidence increased dramatically over the last 50 years and that dengue virus infections expanded to new countries, and from urban to rural settings. Malaysia is one of the countries that experience similar situation. The trend of dengue incidence in Malaysia has continued to increase since 2001 until 2014. In 2001, the dengue incidence rate (IR) was 72 cases in 100,000 population and progressively increased to 361 cases in 100,000 populations in 2014. There were temporary decrease of the incidence rate in 2011 and 2012 to 69.9 and 76 cases per 100,000 populations. Despite the close monitoring and continuous efforts from the Ministry of Health and Municipals to conduct the prevention and control activities, the number of dengue cases continues to increase due to multiple uncontrolled factors. There are at least five major factors that influence the transmission of dengue disease which include the dengue virus, the human as the host, the environmental condition such as cleanliness, the vectors and its behavior and the climate change. Due to these multiple factors that influence the disease pathophysiology and transmission of dengue virus, the control of mosquito-borne viral infection is very challenging and different from managing other infectious diseases. In addition, the rapid urbanization, population growth and human behavior together with international travel, making the control of dengue transmission even more difficult. Based on the circumstances, Ministry of Health Malaysia has implemented the intergrated strategy for dengue prevention and control program in the National Dengue Strategic Plan (NDSP) since 2011. There are seven strategies included in the NDSP which is strengthening of the dengue surveillance, practicing intergrated vector management, emphasizing on the dengue case management, social and community mobilization towards the prevention activity, ensuring rapid response in managing the dengue outbreak and developing new innovative method through dengue research. Most of the factors that contribute to the occurrence of dengue cases are difficult to be controlled and these leave with only manipulation and intervention with the environment, vector control and changing the human behavior for the prevention and control of dengue.
\end{abstract}

Keywords: Dengue incidence, contributing factors, prevention and control strategies, Malaysia.

\section{INTRODUCTION}

\section{Global Burden of Dengue}

Dengue fever has become a global public health problems, WHO has estimated $50 \%$ of world population are at risk and it will continue to rise. Not only the number of cases has increased but the geographical distribution of dengue has also expanded. From the WHO reports, there were more than 100 countries have experienced the dengue epidemics currently compared to only nine countries

\section{Corresponding author:}

Dr. Rose Nani Mudin

Public Health Physician

Head of Vector Borne Disease Sector,

Ministry of Health Malaysia

Email:drrose@moh.gov.my before 1970s. Dengue fever is a vector borne disease and resides in tropical and subtropical countries. It has been an urban area problem in most countries been affected by dengue fever.

\section{DENGUE IN MALAYSIA}

The first dengue fever outbreak in Malaysia was recorded in 1962 from Penang with 41 cases and 5 deaths were reported. Subsequently, a series of outbreak reported in 1973 with 1,487 cases with 54 deaths, 1974 with 2,200 cases with 104 deaths and 1982 with 3,006 cases reported including 35 deaths. Since year 2000, the number of dengue cases and incidence rate (IR) continue to increase with the highest ever reported in 2014. A total of 108,698 cases were reported which is equivalent to IR of 361.1 cases in 100,000 populations in that year (Figure 1 and 2). Fifty six percent $(56 \%)$ of the cases contributed by Klang Valley in which $49 \%$ were from Selangor and $7 \%$ from Federal Territory of Kuala Lumpur-Putrajaya. In the period of 2000 to 2014, the number of reported dengue cases was between 7,103 and 108,698 cases per year and the annual incidence rate range from 31.6 to 361.1 cases per 100,000 populations. All age group were affected with dengue infection and the 


\section{IMJM}

most vulnerable were among the productive aged group between 15 to 49 years old. All ethnic groups are also at risk of being infected. The case fatality rate has reduced from $0.62 \%$ in the year 2000 to $0.2 \%$ in the year 2014 .

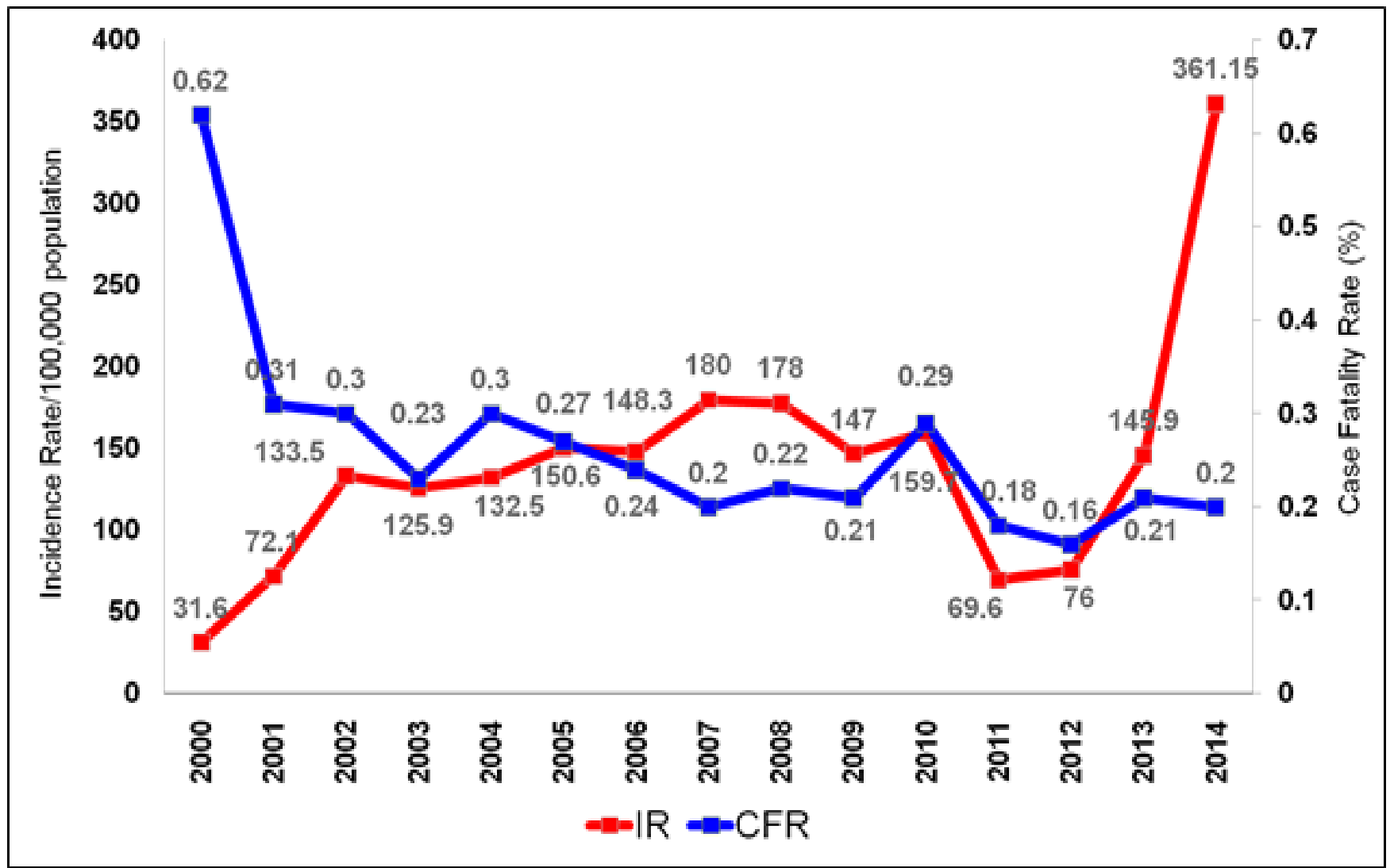

Figure 1. Dengue Incidence Rate and Case Fatality Rate for Malaysia, 2000-2014

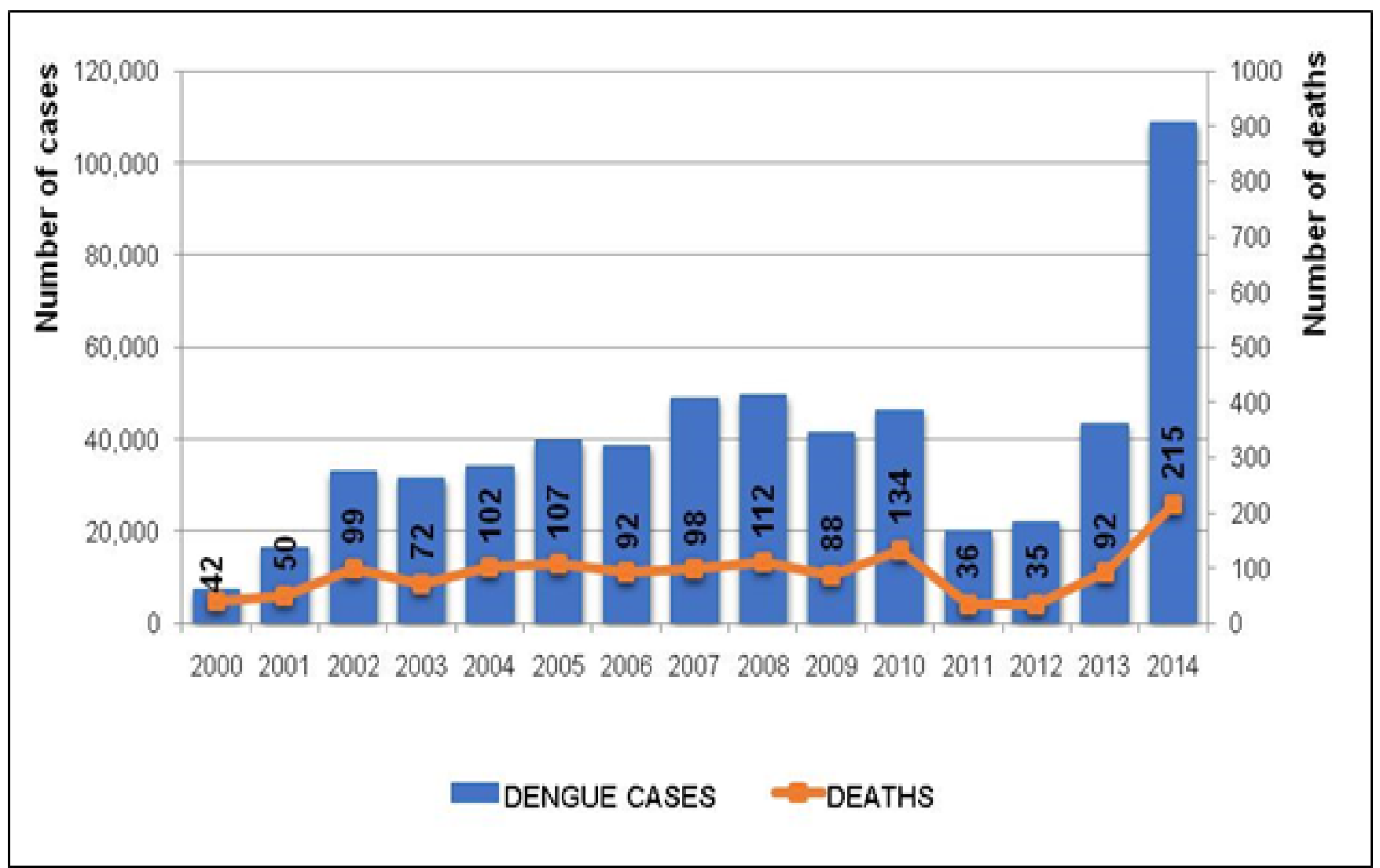

Figure 2. Number of Dengue Cases and Deaths for Malaysia, 2000-2014 


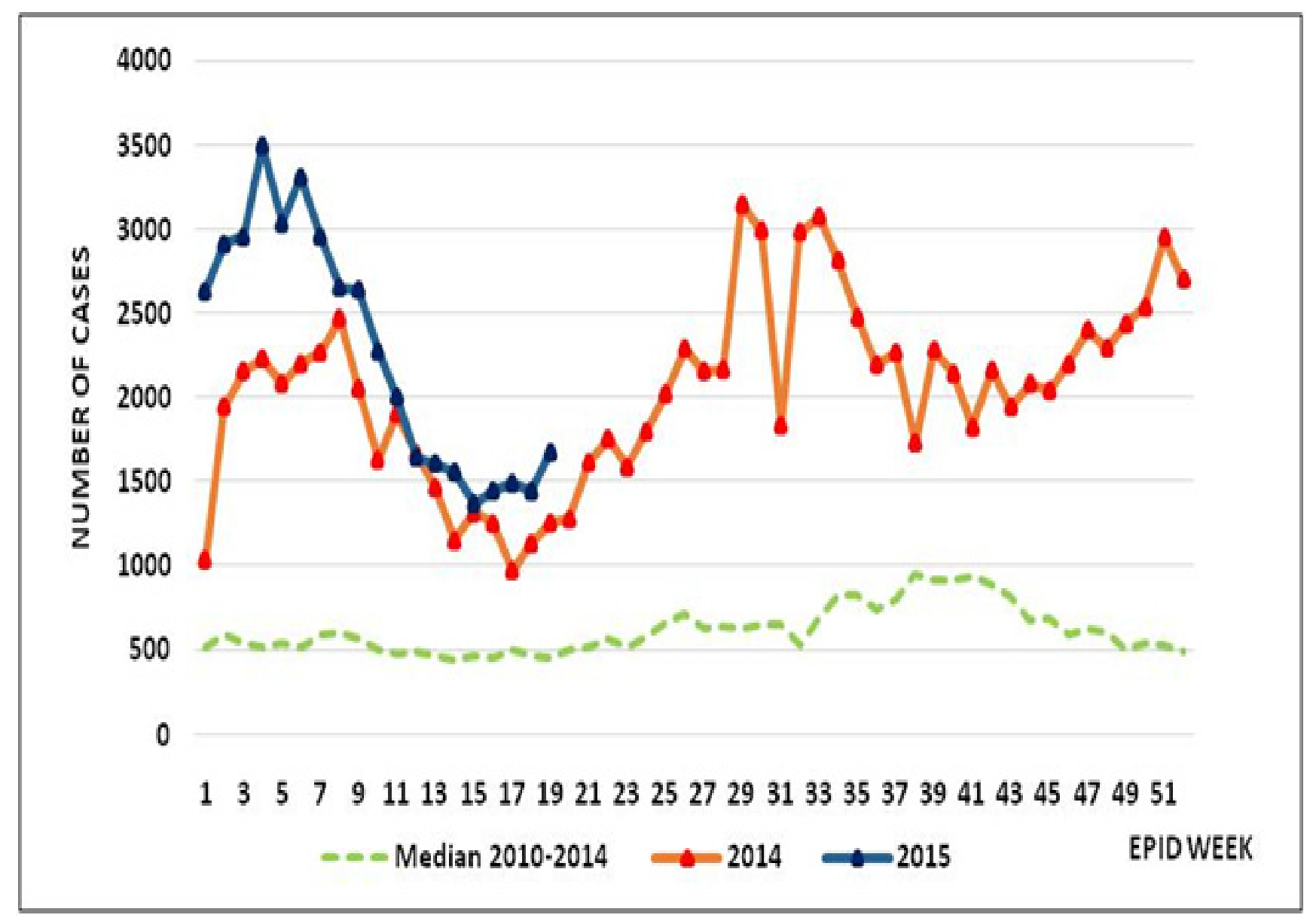

Figure 3. Trend of Dengue Cases by Week in Malaysia

$\mathrm{Bi}$-seasonal peak of the dengue epidemic was observed in 2014 and this pattern was unusual compared to the five years median trend of previous years. It is also observed that the seasonal peak of the epidemic occurred at different month compared to 2013. In 2014, the bi seasonal epidemic peak happened in the month of January to March and July to September. However in 2013, the epidemic peak occurred only from October until December. The seasonal trend of dengue in Malaysia is complex and changes in the pattern of epidemic peak could be the influence of global climate change in this region.

\section{Dengue Serotype in Malaysia}

Ministry of Health $(\mathrm{MOH})$ Malaysia has established the Dengue virus surveillance since 1990s from various laboratories in the main hospitals and Institute of Medical Research. In 2009, a structured virus surveillance system called "Dengue Virus Surveillance System (DVSS)" was established and the collections of samples were expended to all the 14 states on weekly basis.

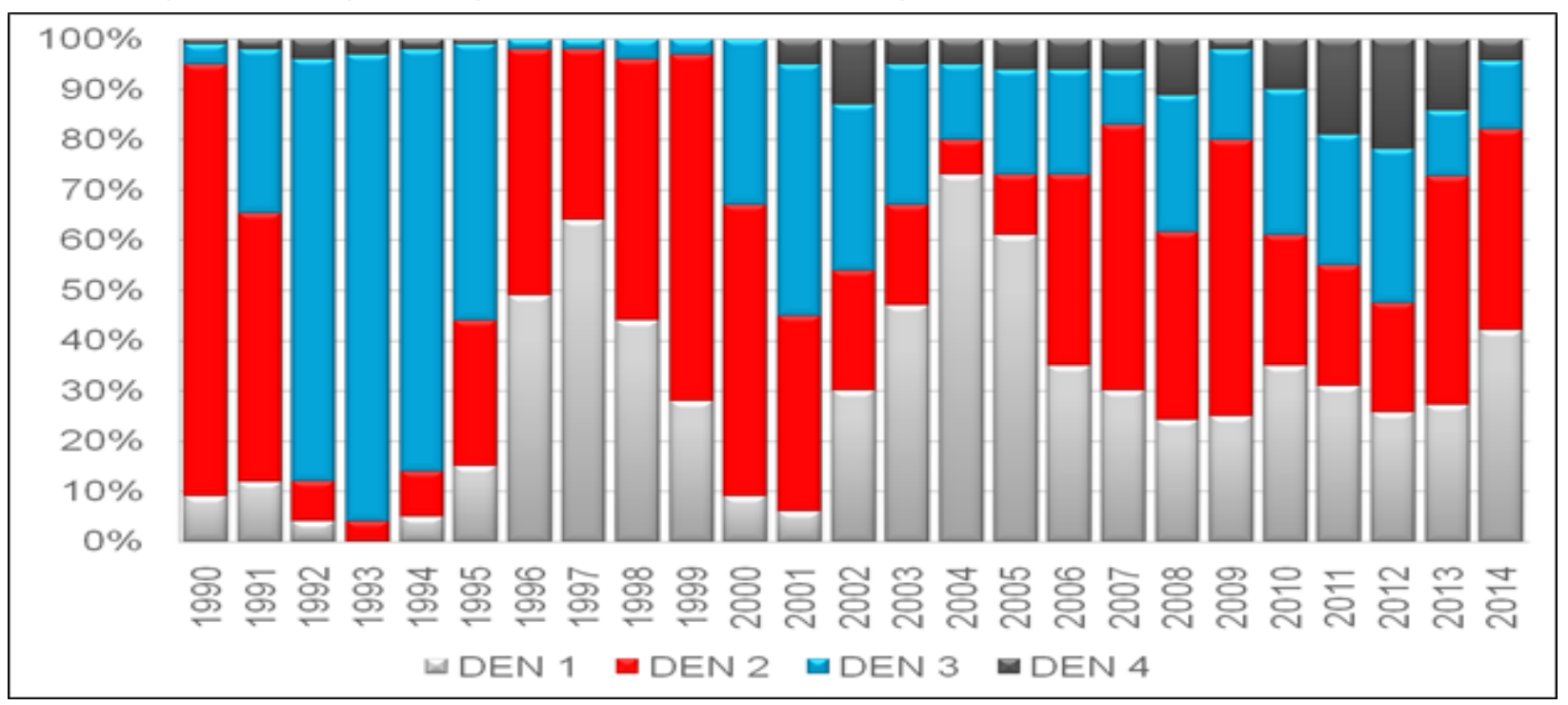

Figure 4. Dengue Virus Serotype in Malaysia, 1990-2014 
All four dengue virus serotypes (DENV-1, DENV-2, DENV-3, and DENV-4) are found in Malaysia and the predominant serotype changed from year to year. For example in between 1992 to 1995 the predominant serotype was DEN 3 and beginning 1996 the predomi- nant serotype changed between DEN 1, DEN 2 and DEN 3. A small proportion of DEN 4 was found in some states. It is also observed that the predominant serotype varies between the states.

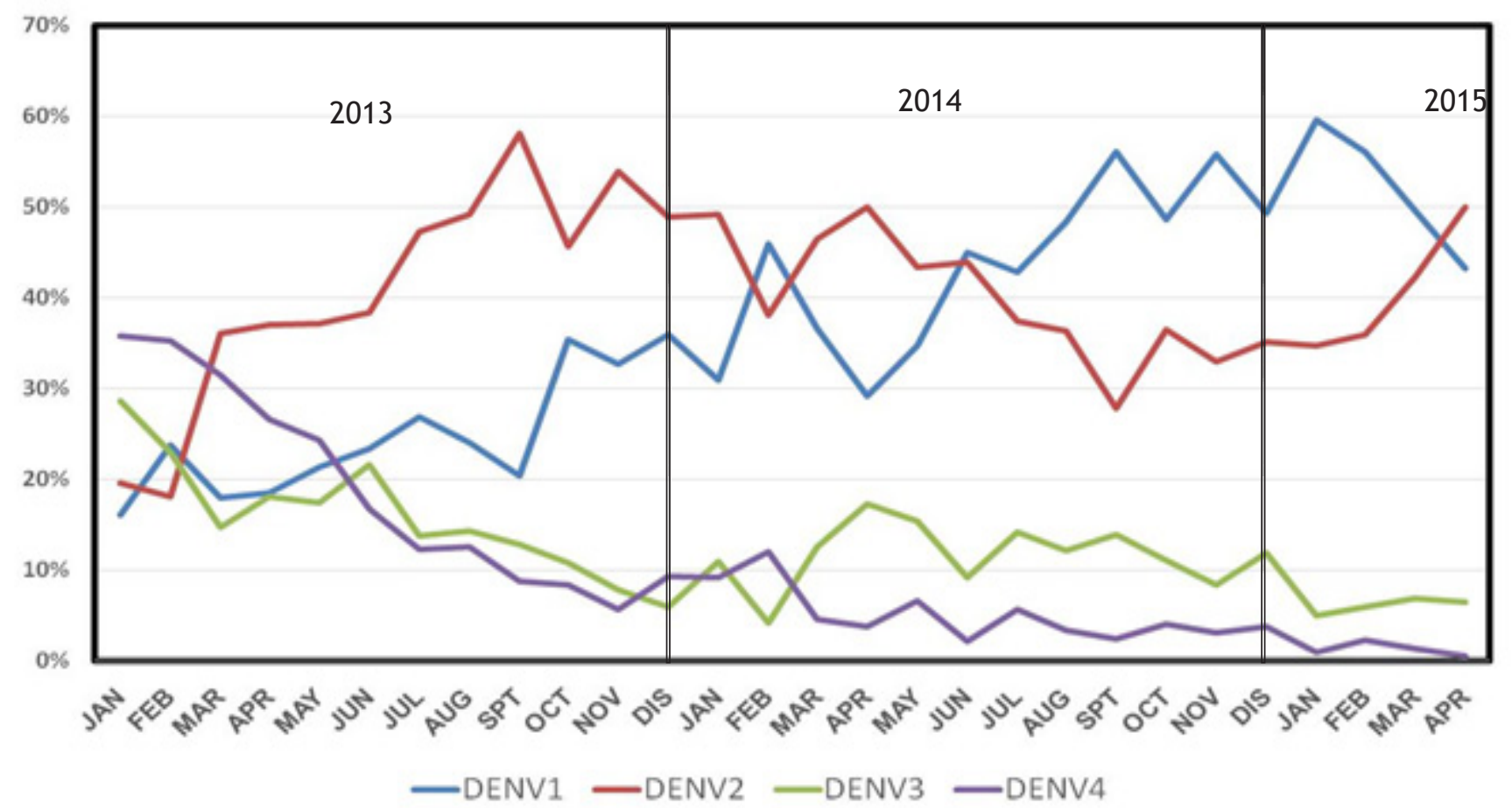

Figure 5. Dengue Serotype Shift

From the serotypes surveillance, it showed that there was a serotype shift from DEN 4 to DEN 2 occurred in March 2013 and followed by a surged of cases starting in September 2013 until January 2014. Once again, Malaysia experienced twice of serotype shifts which were in March and Jun 2014. Subsequently, the epidemic peak followed from July until September in that particular year. The $\mathrm{MOH}$ epidemiology surveillance monitoring showed that 4 to 6 months after the serotype shifts, it is more likely that a surged of dengue cases to occur. Changes of the dengue serotype (serotype shift) in the community led to an increase of dengue cases and deaths due to reduction in the herd immunity to the new virus serotypes.

\section{Factors Contributing To Increasing Dengue Incidence}

A part from the serotypes shift, other contributing factors also play major role in the occurrence of epidemic peak in Malaysia. Changes in the climate such as temperature rising, increased rainfall, and relative humidity are the most influential driving forces of dengue transmission and intensity in this country. During Malaysia's rapid industrialization and economic growth in the past several decades, massive infrastructure development resulted in spread of the disease. A few studies have shown that the continuous process of urbanization has resulted in increased incidence of dengue in Malaysia. Human movement are more widespread, especially for patients who have been infected with dengue virus could easily spread the virus to another place when theys visit new places.
The occurrence of dengue cases is also contributed by the high Aedes breeding index. Poor environmental cleanliness due to habit of littering in the community and in appropriate solid waste disposal is the main issue in many countries including Malaysia. From the $\mathrm{MOH}$ analysis, it shows that polisterine food container, plastics bottles and tyres contribute the highest percentage of breeding places. The average Aedes Index (AI) for 2015 ranged between 1.5 to $2 \%$.

\section{Integrated Management Strategy In Dengue Prevention and Control}

Due to the various factors that influence the transmission of dengue virus, the control of mosquito-borne viral infection is very challenging and different from managing other infectious diseases. In principles, all the factors contributing to the disease transmission must be well controlled. However most of the factors are difficult to be controlled and these leave with only manipulation and intervention with the environment cleanliness, vector control and changing in the human behavior. Based on this fact, Ministry of Health has implemented the Integrated Management Strategy for dengue prevention and control program since 2011.

To deal with the dengue epidemic surge, various efforts have been made by Ministry of Health through integrated actions involving 6 other ministries. In 2013, an "Ops Mega" campaign was launched in 4 districts of Selangor state, to curb the rise of dengue cases. The nationwide consented efforts managed to 
reduce $60 \%$ of the total number of cases for the country within 3 months. However, the biggest challenge is to sustain the preventive activity among the community and lower the number of dengue cases.

Since 2014, integrated enforcement activities between the Ministry of health, the Construction Industry Board (CIDB) and the Department Of Safety And Employment has been carry out regularly at the district and state level. Although many of this construction sites have been closed due to massive Aedes breeding, there is still a lot more construction site need to be inspected with the limited human resources in the Ministry of Health.

\section{CONCLUSION}

The prevention of dengue disease require a participation or involvement of the community, together with other agencies including private and public sectors. The role of community and other agencies in ensuring the "search and destroy" activities for Aedes breeding places need to be carried out weekly. It is the most important element in the succes of controlling dengue.

\section{REFERENCES}

All statistics and datas quoted in this article are from the authors work and the Ministry of Health Malaysia. 